\title{
The Effect of Shot-Peening Treatment on Microstructure and Corrosion Behavior of Closed-Cell Aluminum Foam
}

\author{
Chengwu Zhang1, Yongdong $\mathrm{He}^{1 *}$, Yanhua Chen ${ }^{1 *}$, Yongliang $\mathrm{Mu}^{2}$, Fengjun Zhao' ${ }^{1}$ Xiaochun $\mathrm{Li}^{1}$ \\ ${ }^{1}$ School of Physics Science and Technology, Xinjiang University, Urumqi, China \\ ${ }^{2}$ School of Metallurgy, Northeastern University, Shenyang, China \\ Email: *hydongq@126.com, *chenyanhua97@163.com
}

How to cite this paper: Zhang, C.W., He, Y.D., Chen, Y.H., Mu, Y.L., Zhao, F.J. and Li, X.C. (2017) The Effect of Shot-Peening Treatment on Microstructure and Corrosion Behavior of Closed-Cell Aluminum Foam. World Journal of Engineering and Technology, 5, 89-98.

https://doi.org/10.4236/wjet.2017.54B010

Received: September 13, 2017

Accepted: October 9, 2017

Published: October 12, 2017

\begin{abstract}
Closed-cell aluminum foam was shot peened at different processing time $(0 \mathrm{~s}$, $5 \mathrm{~s}, 10 \mathrm{~s}$, and $20 \mathrm{~s}$ ), the intensity was the $0.12 \mathrm{mmA}$. The X-ray diffraction results showed that the reflections became weakened obviously with the shot peened time increased. Combined with Popa model and lognormal distribute model, the surface microstructure of closed-cell aluminum foam was investigated by using the Rietveld whole pattern fitting analysis method. The results revealed that domain size and microstrain fluctuated along different reflection directions after shot peened, which attributed to the random and anisotropic deformation direction. With the shot peened processing time prolonged, a decrease in domain size and an increase in microstrain were also observed. Moreover, the corrosion behavior of closed-cell aluminum foam was studied by weight-loss test. The results indicated that corrosion properties of specimen subjected to shot peened processing was better than the unpeened specimens.
\end{abstract}

\section{Keywords}

Closed-Cell Aluminum, Shot Peened, Microstructure, X-Ray Diffraction Linear Analysis, Corrosion Behavior

\section{Introduction}

Aluminum foam, a kind of new function-structure materials, has a development application prospect in many aspects. According to the cell space structure, the aluminum foam can be classified as closed cell aluminum foam (CAF) and open aluminum foam. The particularity of foam metal structure endows these mate- 
rials with many excellent properties, such as light-weight, thermal-insulation, energy-absorbing, permeation performance etc. Thus, the foam aluminum has got extensive application in different fields [1] [2] [3] [4].

Recently, great progress has been made in the CAF corrosion resistance. Nima Movahedi et al. [5] reported that the corrosion resistance of closed-cell Al-A356 alloy foam can be enhanced by the plasma electrolytic oxidation treatment. Zhang Yongming et al. [6] studied the influence of rare earth elements (REE = $\mathrm{La}, \mathrm{Y}, \mathrm{Ce}$ ) on corrosion behavior of CAF, and found that adding proper amount of REE can improve the corrosion resistance of CAF. However, this approach would be greatly restricted by the relatively expensive REE. Shot peening (SP) was a mechanical surface treatment method, which not only can effectively enhance fatigue strength and fatigue life, but also significantly improve the corrosion behavior of material [7]. However, up to now, the effect of SP treatment on the surface-layer microstructure and property of CAF has been rarely reported.

In this paper, the SP treatment was applied to CAF. The surface information of CAF at different time has been obtained by X-ray diffraction (XRD), and the evolution of microstructure induced by SP was further investigated by Rietveld whole pattern fitting analysis method. In addition, the corrosion behavior and mechanism of CAF was discussed.

\section{Experimental Procedure}

\subsection{Specimens Preparation}

CAF was fabricated by melt-foaming method at the Xinjiang University of China. The amount of thickening agent (high purity $\mathrm{Ca}$ about $3 \mathrm{wt} \%$ of the melt mass) was added to the molten aluminum (commercial purity $\mathrm{Al}$ ), and foaming agent (high purity $\mathrm{TiH}_{2}, 1.5 \mathrm{wt} \%$ of the melt mass) was added. Meanwhile, by the agitation of the foaming agent were dispersed and release gas, after a certain time of heat preservation, the molten aluminum foaming after cooling solidification in the mold was the CAF. The apparent densities of the foams were about $0.67 \mathrm{~g} \cdot \mathrm{cm}^{-3}$, cell size was approximately $3-7 \mathrm{~mm}$, porosity was $74 \%$, and selecting the same pore structure of CAF specimens was cut into $18 \times 11 \times 4.5 \mathrm{~mm}$ by the electrical discharge machining. SP pellets used in this study were conditioned carbon steel cut wire shot (CCW14) balls with the diameter of $\sim 0.30 \mathrm{~mm}$ and the average hardness of pellets was $610 \mathrm{Hv}$. The specimens were shot-peened on one side with the surface coverage of more than $100 \%$. SP intensity was set at 0.12 $\mathrm{mmA}$, and SP time was $0 \mathrm{~s}, 5 \mathrm{~s}, 10 \mathrm{~s}$ and $20 \mathrm{~s}$, respectively. Specimens processing status and number were shown in Table 1.

Table 1. Specimens processing status and time.

\begin{tabular}{cc}
\hline Specimens & Peening's time $(\mathrm{S})$ \\
\hline NP & 0 \\
SP1 & 5 \\
SP2 & 10 \\
SP3 & 20 \\
\hline
\end{tabular}




\subsection{Corrosion Test}

The corrosion resistance of the specimens was obtained by Weight-loss testing [8]. Using total immersion method, the specimens were corroded for 24 hours at the room temperature $\left(20^{\circ} \mathrm{C} \pm 1^{\circ} \mathrm{C}\right)$, with simulate seawater $(3.5 \% \mathrm{NaCl}+1 \%$ $\mathrm{H}_{2} \mathrm{O}_{2}$ in aqueous solution, $\mathrm{PH}=5$ ), and then cleaned with $5 \% \mathrm{HNO}_{3}+1 \% \mathrm{~K}_{2} \mathrm{Cr}_{2} \mathrm{O}_{7}$ solution. After ultrasonic cleaned in anhydrous ethanol and deionized water washing, the product was dried, and then weighed. The same weight-loss testing experiment was repeated three times to reduce error. The mass loss rate $A$ was calculated according to the following equation:

$$
A=\frac{w_{0}-w_{1}}{w_{0}} \times 100 \%
$$

where $w_{0}$ was the initial mass of the specimens before the corrosion and $w_{1}$ was the finial mass of the specimen after the corrosion.

\subsection{Characterization Methods}

Prior to and after the weight-loss testing, the weight of specimen was measured accurately using the SARTORIUS CP225D analytic balance with the accuracy of $10^{-4} \mathrm{~g}$. The surface structure analysis of specimens were performed by Bruker D8 Advanced $\mathrm{X}$-ray diffractometer with $\mathrm{Cu}-\mathrm{K}_{\alpha}$ radiation $(\lambda=0.1542 \mathrm{~nm})$, using step-scan ways with $2 \theta$ ranging from $30^{\circ}-120^{\circ}$. The microstructure was further analyzed by the XRD linear analysis methods. The surface topography was characterized by scanning electron microscope (SEM), and the surface chemical composition of specimens was conducted by the energy dispersive X-ray spectroscopy (EDS).

\section{Results and Discussion}

\subsection{The Influence of SP Processing Time on Surface Microstructure of CAF}

Figure 1 shows the evolution of the X-ray diffraction patterns with the SP processing

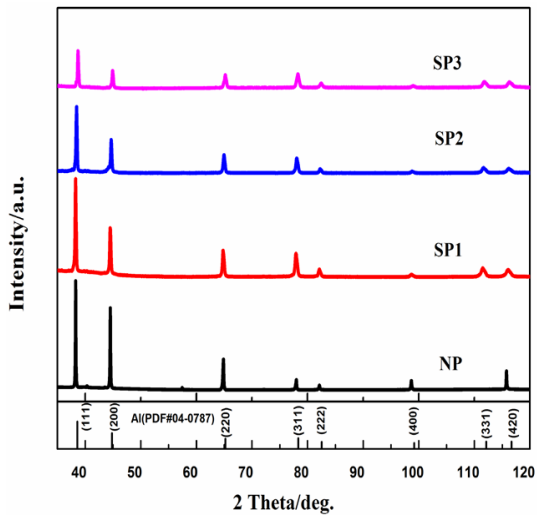

(a)

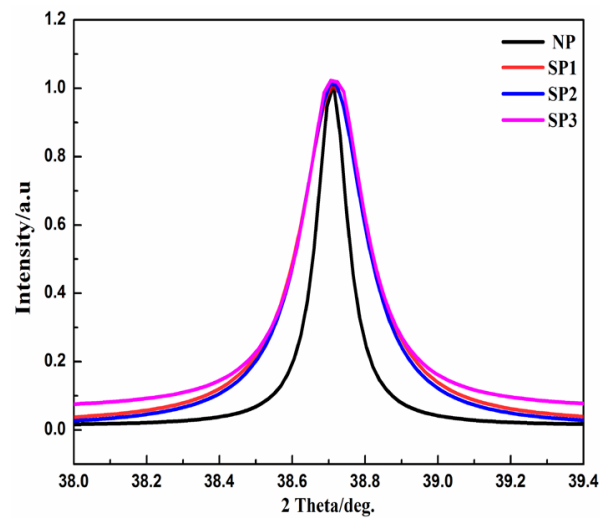

(b)

Figure 1. (a) XRD patterns of SP specimens; (b) The normalized XRD pattern, SP intensity $0.12 \mathrm{mmA}$. 
time increasing. It can be seen that the diffraction peaks of $\mathrm{Al}$ (PDF\#04-0787) was clearly, and there was no other new phase after SP. With the increasing of SP processing time, the intensity of the diffraction peak was significantly weakened and the breath of [111] diffraction peak was broaden obviously. This phenomenon can be explained by the deformation mechanism induced by SP. During the process of SP, a great number of pellets impact the surface of the material from different directions, which causes elastic and plastic deformation at and near surface layer of the specimens [9]. Thus, the domain was refined and the orientation randomization of domain was enhanced, due to the randomization of deformation direction in the surface material after SP.

\subsection{Rietveld Whole Pattern Fitting the Linear Analysis of SP Specimens}

In order to further obtain the microstructure information of the CAF, the Rietveld whole pattern fitting method was applied with use Materials Analysis Using Diffraction (MAUD) software. The fitting result of the SP3 specimen was showed in Figure 2. It can be seen that, the fitting line and the observed diffraction line were in good agreement, and the weighted profile $R_{w}$ was $6.7 \%$. The XRD patterns of NP, SP1, and SP2 specimen were also fitted, and the $R_{w}$ of which were $8.37 \%, 8.24 \%$ and $8.37 \%$, respectively. The $R_{w}$ values were less than $10 \%$, and which suggested that the results of fitting processing were reliable.

XRD linear analysis method was one of the most effective methods to characterize the microstructure of materials. X-ray diffraction pattern refers to the diffraction intensity with the change curve of the diffraction angle.

$$
d^{*}=2 \sin \theta / \lambda
$$

The observed diffraction linear specimens $h(x)$ can be expressed as instrument linear $g(y)$ and physical linear $f(x-y)$ convolution of specimens. The reason for instrument line broadening were that: 1) The wavelength of the incident X-ray was not completely monochromatic; 2) X-ray light source, specimen, receiving slit was not completely meet the focused condition; 3 ) The incident

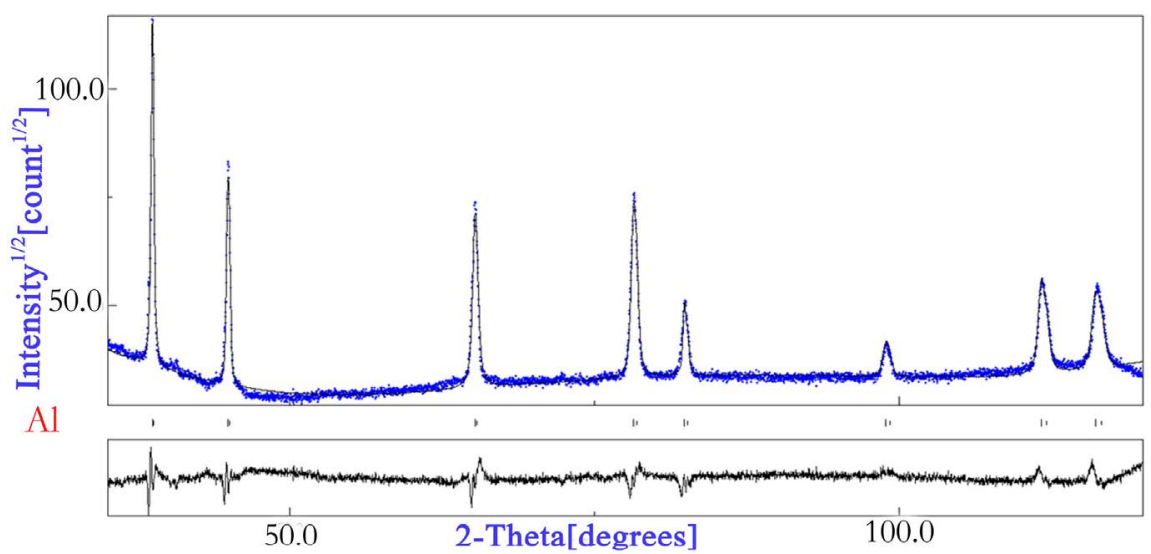

Figure 2. Observed and calculated XRD patterns of SP3, shot peening intensity was 0.12 mmA. 
X-ray was not completely parallel etc. The mainly factors affected the physical linear microstructure included: domain size, strain and crystal structural defects responsible for line etc., these factors responsible for line broadening effects was independently.

$$
h(x)=\int_{\infty}^{\infty} g(y) f(x-y) d y
$$

$f(x-y)$ function was related to grain size and strain. Direction-dependent crystallite size and strain models were put forward by Popa [10] [11].

In this model, the normalized peak profile was:

$$
V_{H}(z)=\int d\left(\Delta z^{\prime}\right) G_{H} d\left(\Delta z^{\prime}\right) L_{H}\left(z+\Delta z^{\prime}\right)
$$

The Gaussian component $G_{H}$ represents the strain effect, and the Lorentzian component $L_{H}$ represents the size effect. In terms of the integral breadths $\beta_{G H}$, $\beta_{L H}$ these functions were:

$$
\begin{gathered}
G_{H}(z)=\beta_{G H}^{-1} \exp \left(-\pi z^{2} / \beta_{G H}^{2}\right)^{-1} \\
L_{H}(z)=\beta_{L H}^{-1}\left(1+\pi^{2} z^{2} / \beta_{L H}^{2}\right)^{-1}
\end{gathered}
$$

For the constant-wavelength diffraction method, $z=2 \theta$ and the quantities $\beta_{G H} \quad \beta_{L H}$ were shown as follows.

$$
\begin{gathered}
\beta_{G H}=2 \tan \theta_{H}\left(2 \pi\left\langle\varepsilon_{h k l}^{2}\right\rangle\right)^{1 / 2} \\
\beta_{L H}=2 \lambda /\left(3\left\langle R_{h}\right\rangle \cos \theta_{H}\right)
\end{gathered}
$$

According to the obtained diffraction line parameters and based on the follow principles, direction-dependent domain size and microstrain of the CAF has been obtained from the broadening of diffraction peaks. For face-centered cubic $(f, c, c)$ crystal, The space group was Fm3m, domain size and microstrain expression shown as below:

$$
\begin{aligned}
& <D>=D_{0}+D_{1} K_{4}^{1}(x, \varphi)+D_{2} K_{6}^{1}(x, \varphi)+D_{3} K_{6}^{1}(x, \varphi)+\ldots \\
& <\varepsilon^{2}>E_{H}^{4}=E_{1}\left(h^{4}+k^{4}+l^{4}\right)+2 E_{2}\left(h^{2} k^{2}+h^{2} l^{2}+k^{2} l^{2}\right)
\end{aligned}
$$

The results obtained via XRD linear analysis methods were showed Figures 3-6. It can be seen from Figure 3 that the FWHM increased with the SP time increasing which indicated that the domain was refined and the microstrain was increased after SP.

Figure 4 shows the domain size varies with the SP time. It was clear that the domain of major peaks (111) and (200) was refined obviously after $5 \mathrm{~s}$-SP, and then only a slight variation can be discerned with the SP time prolonged. The results reveal that $5 \mathrm{~S}$ was the optimal processing time.

The variation trend of microstrain was shown in Figure 5, which was inversed of Figure 4. An interesting phenomenon can be found that, the microstrain decreased firstly and then increased with the SP time prolonged. This phenomenon may be due to CAF special porosity structure and property. During the SP processing, 


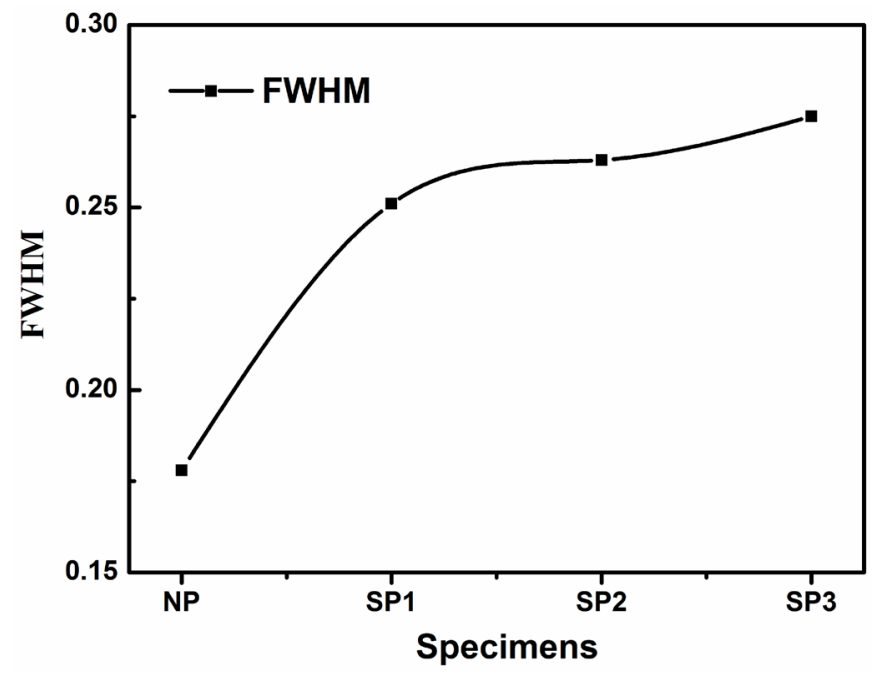

Figure 3. Variation of the FWHM in NP, SP1, SP2, and SP3.

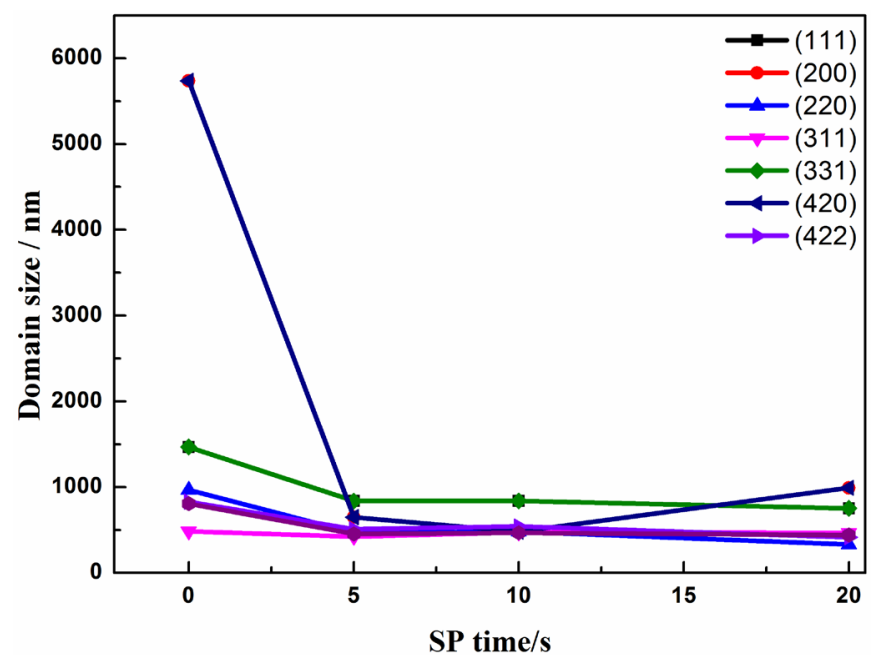

Figure 4. Direction-dependent domain size of CAF surface with the change of shot peening time.

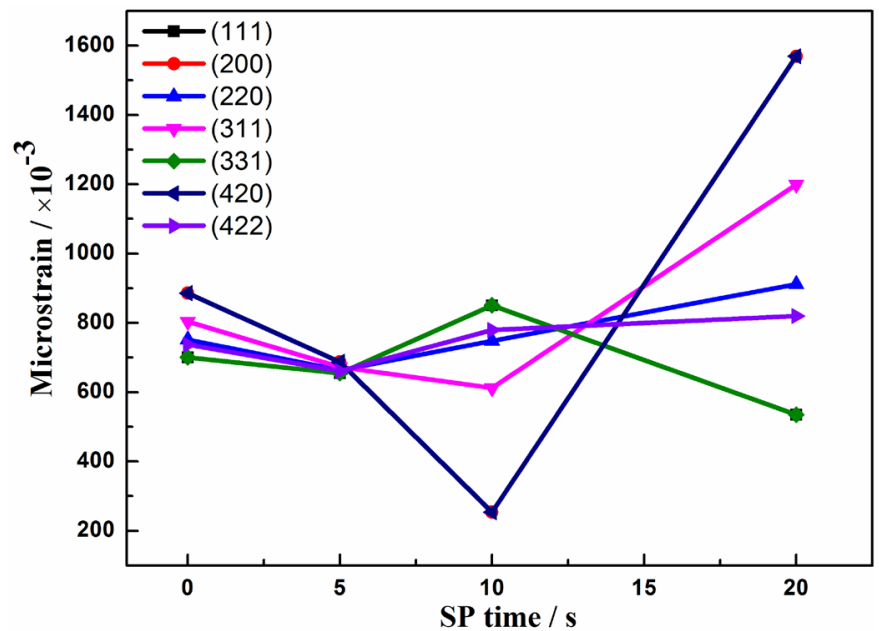

Figure 5. Direction-dependent microstrain of CAF surface with the change of SP time. 


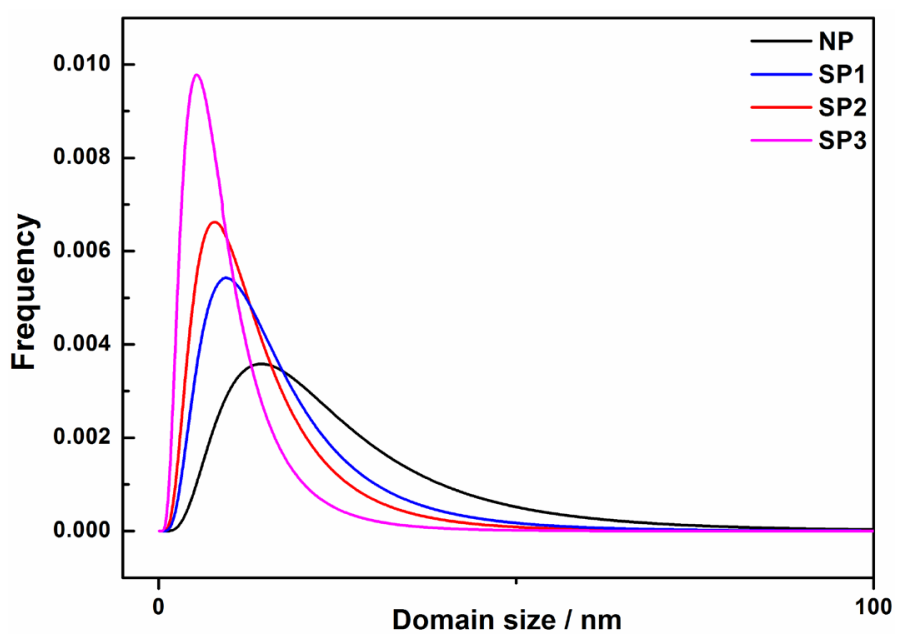

Figure 6. Frequency distribution of domain size of specimens NP, SP1, SP2, SP3.

the deformation of cell structure and shear collapse of CAF was random, and the magnitude of the stress was not affected.

The average domain size along with SP time was characterized by the lognormal distribution model, and results were shown in Figure 6. As the processing time increased, the average domain sizes decreased and domain sizes lognormal distribution curve obviously narrowed [12].

\subsection{The Corrosion Resistance of CAF}

The effect of SP treatment on corrosion resistance of CAF was also discussed. It can be seen from Figure 7 that the mass loss rate of the shot-peened specimens was smaller than that of the unpeened specimen. It should be clear that SP can effectively improve the corrosion resistance. Compared with the SP1, SP2 and SP3 specimens, the mass loss rate of SP1 was the smallest, which indicated that the corrosion resistance of SP1 was best. This experiment results showed that 5 seconds was the optimal processing time, which was consistent with the Rietveld fitting results. The surface nanocrystallization has been induced, and composition was more homogeneous than unpeened specimens [13]. The difference of electrochemical decreased in grain interior and grain boundary, and local corrosion on interface diminished. Meanwhile, the residual compressive stresses (RCS) on the surface would inhibit corrosion crack initiation and propagation [14]. And the ability of corrosion resistance was improved.

The surface morphologies were observed by SEM from Figures 8(a)-(d). Figure $8(\mathrm{c})$ showed the CAF cell local area appear perforated hole, this phenomenon made pore connectivity increased, the materials and corrosive medium real contact area increased, the corrosion resistant decreased. Meanwhile, Figure 8(d) showed that microcracks were induced by SP .This phenomenon suggests with the processing time increasing, the damage and failure of the surface materials were serious. The existence of perforated hole and microcrack decreased the corrosion resistance of CAF. In all, effect the surface nanocrystallization and 


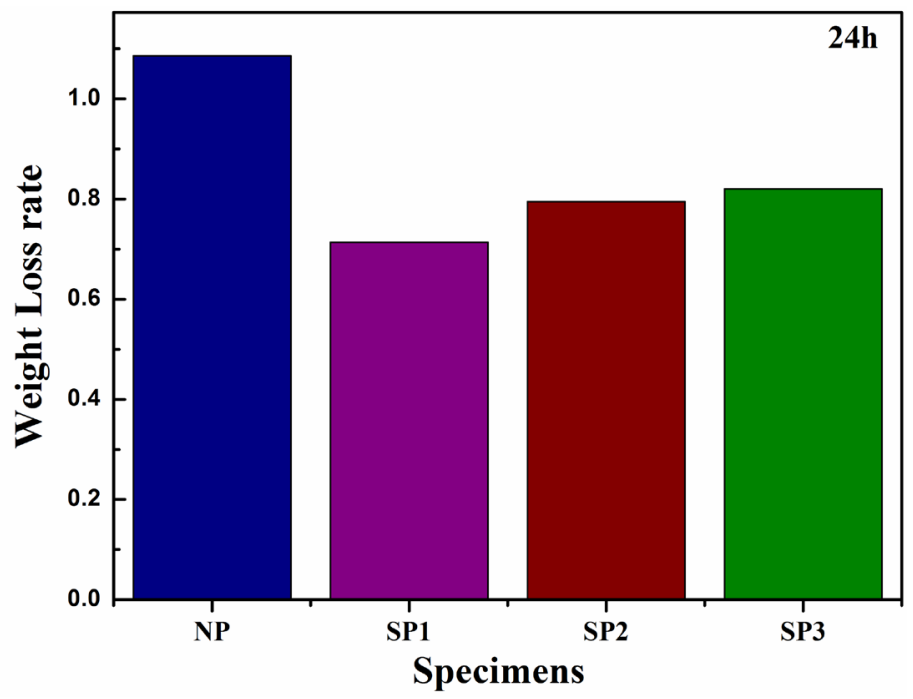

Figure 7. The experimental results of NP, SP1, SP2, SP3, Corrosion 24 hour.

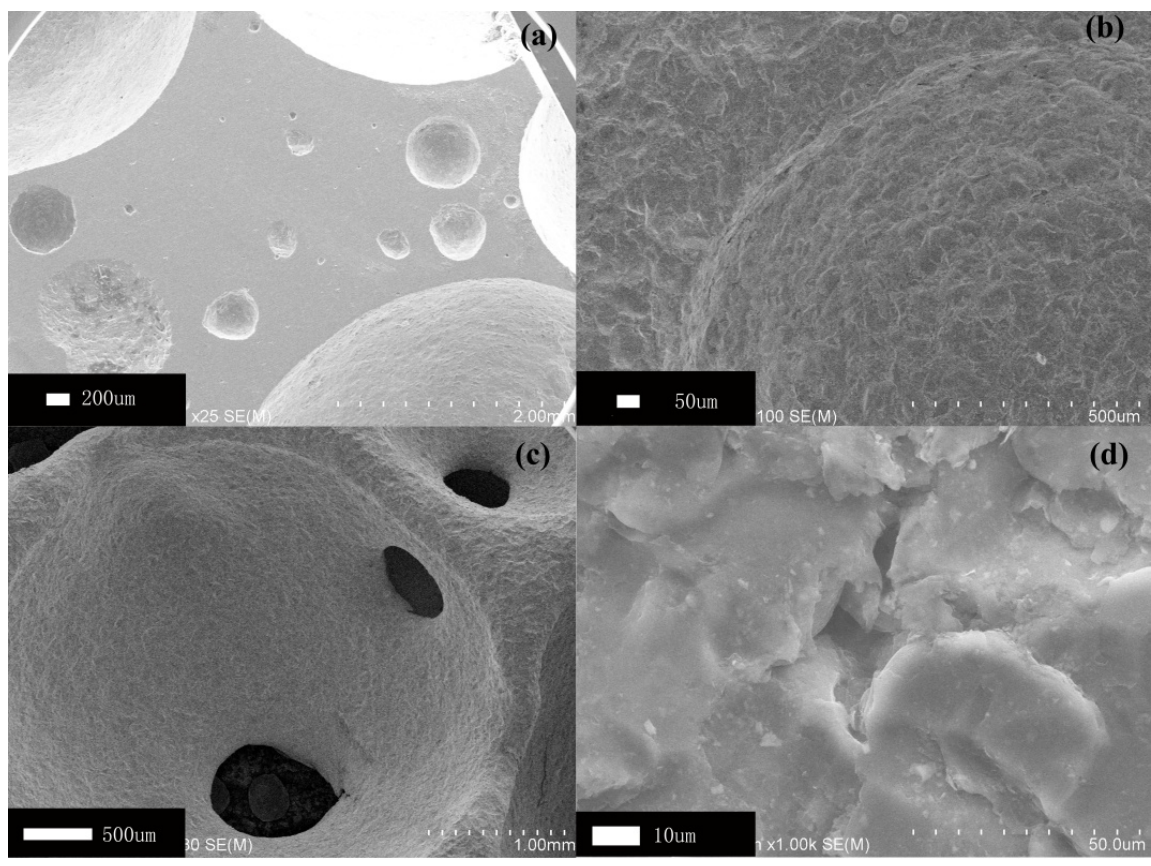

Figure 8. The SEM images of specimen's surface topography: (a) NP specimen; (b) Shot-peened specimen; (c) Perforation phenomenon of specimen; (d) Microcrack of the specimen

compressive residual stress field still remain dominate, which were much better than disadvantage brought by the surface damage of the CAF.

The chemical composition of specimen's surface was analyzed by X-ray spectroscopy (EDS). The element contents were given in Table 2. It can be seen from that the major compound of surface materials was aluminium oxide. Compared with atomic percentage of NP and SP1, which of the elements $\mathrm{O}$ increased so that more aluminium oxides were generated. It was meaning that large fraction of the non-equilibrium grain boundaries furnished the specimens by SP, with 
Table 2. Chemical composition distribution of the specimens surface NP, SP1, SP1 + Corrosion.

\begin{tabular}{ccc}
\hline Specimen & $\mathrm{Al}($ at\%) & $\mathrm{O}(\mathrm{at} \%)$ \\
\hline NP & 85.91 & 04.67 \\
SP1 & 66.10 & 07.77 \\
SP1 + Corrosion & 39.05 & 32.87 \\
\hline
\end{tabular}

more nucleus to form denser nature oxide film than ordinary grain [15]. This denser oxide film could be keeping more stable and relative integral in an oxidative atmosphere. Meanwhile, compared with atomic percentage of the SP1, SP1 and corrosion, the elements $\mathrm{O}$ also increased. It obviously showed that the percentage of the elements $\mathrm{O}$ in the specimens surface SP1 and corrosion was higher than the NP and SP1. It was meaning that corrosion can cause the stress relaxation, and the deep oxidation occurred more easily.

\section{Conclusion}

In this paper, with the following important conclusion being the list: The XRD results showed that the reflection became broaden obviously with the SP time increased. And the nanocrystalline grain of SP specimens was also obtained. The Rietveld whole pattern fitting analysis method results revealed that domain size and microstrain fluctuated along different reflection directions after SP, which attributed to the random and anisotropic deformation direction. Weight-loss test indicated that corrosion properties of CAF subjected to SP processing was better than the unpeened specimens. The Rietveld whole pattern fitting analysis method and experimental results showed that 5 seconds was the optimal processing time.

\section{Acknowledgements}

Financial support from the High-tech development project (201515106) of Xinjiang Uyghur Autonomous Region and Natural Science Foundation of China under Project No. 11264037 was acknowledged.

\section{Foundation Item}

Projects (11264037) supported by the National Natural Science Foundation of China; Project (201515106) supported by high-tech development project of Xinjiang Uyghur Autonomous Region.

\section{References}

[1] Banhart, J. (2005) Aluminium Foams for Lighter Vehicles. International Journal of Vehicle Design, 37, 114-125. https://doi.org/10.1504/IJVD.2005.006640

[2] Banhart, J. (2013) Light-Metal Foams-History of Innovation and Technological Challenges. AdV Eng Mater, 15, 82-111. https://doi.org/10.1002/adem.201200217

[3] Zhao, W.M., Ma, Y.D., Hou, S.P. and Hao, Z.W. (2001) Development Research and 
Application about Foam Metals. Journal of Hebei University of Technology, 30, 50-55.

[4] Wang, P.P. and Yu, Y.H. (2003) Study on the Properties of Foamed Aluminium and Its Applications to Automobiles. Coal Mine Machinery, 11, 46.

[5] Movahedi, N. and Habibolahzadeh, A. (2016) Effect of Plasma Electrolytic Oxidation Treatment on Corrosion Behavior of Closed-Cell Al-A356 Alloy Foam. Mater Lett, 164, 558-561. https://doi.org/10.1016/j.matlet.2015.11.073

[6] Yong, M., Zhang, D. and Ge, D. (2006) Effect of Rare Earth on Corrosion Behavior of Aluminum and Aluminum Alloy Foam. Materials for Mechanical Engineering, $30,19-21+78$.

[7] Al-Obaid, Y. (1995) The Effect of Shot Peening on Stress Corrosion Cracking Behaviour of 2205-Duplex Stainless Steel. Engineering Fracture Mechanics, 51, 19-25. https://doi.org/10.1016/0013-7944(94)00213-2

[8] Zhang, Y.M., He, D.P., Shi, G.D., Chu, X.M., Dai, G. and Wang, H. (2008) Corrosion Resistance and Surface Area of Closed-Cell Aluminum. Materials for Mechanical Engineering, 32.

[9] Chen, Y. and Jiang, C. (2013) Effect of Shot Peening on Surface Characteristics of Ni-Based Single-Crystal Superalloy. Materials Transactions, 54, 1894-1897. https://doi.org/10.2320/matertrans.M2013153

[10] Balzar, D., Audebrand, N., Daymond, M.R., Fitch, A., Hewat, A., Langford, J.I., et al. (2004) Size-Strain Line-Broadening Analysis of the Ceria Round-Robin Sample. $J$ Appl Crystallogr, 37, 911-924. https://doi.org/10.1107/S0021889804022551

[11] Popa, N. (1998) The (hkl) Dependence of Diffraction-Line Broadening Caused by Strain and Size for All Laue Groups in Rietveld Refinement. J Appl Crystallogr, 31, 176-180. https://doi.org/10.1107/S0021889897009795

[12] Xie, L., Feng, Q., Wen, Y., Wang, L., Jiang, C. and Lu, W. (2016) Surface Microstructure Characterization on Shot Peened $(\mathrm{TiB}+\mathrm{TiC}) / \mathrm{Ti}-6 \mathrm{Al}-4 \mathrm{~V}$ by Rietveld Whole Pattern Fitting Method. J Mater Res, 31, 2291-2301. https://doi.org/10.1557/jmr.2016.256

[13] Wang, T., Yu, J. and Dong, B. (2006) Surface Nanocrystallization Induced by Shot Peening and Its Effect on Corrosion Resistance of 1Cr18Ni9Ti Stainless Steel. Surf Coat Technol, 200, 4777-4781. https://doi.org/10.1016/j.surfcoat.2005.04.046

[14] Zagar, S., Grum, J. (2015) Roughness, Residual Stresses and Pitting Corrosion Effect on Shot Peened AA 7075. [Hrapavost, zaostala naprezanja i tockasta korozija na sacmarenom AA 7075.] Tehnicki Vjesnik-Technical Gazette, 22, 1589-1596.

[15] Abdulstaar, M., Mhaede, M., Wagner, L. and Wollmann, M. (2014) Corrosion Behaviour of Al 1050 Severely Deformed by Rotary Swaging. Materials \& Design, 57, 325-329. https://doi.org/10.1016/j.matdes.2014.01.005 